\title{
LA INVENCIÓN COLECTIVA ANTE CIRCUNSTANCIAS ADVERSAS
}

\section{THE COLLECTIVE INVENTION FACED TO SOME ADVERSE CIRCUMSTANCES}

\author{
Karina Benito*
}

\section{Resumen}

En el artículo se presentan los avances de un proyecto de investigación doctoral en ciencias sociales en la Universidad de Buenos Aires. A partir de una metodología cualitativa se exploraron espacios, clubes y centros culturales en la ciudad de Buenos Aires, desde el retorno de la democracia hasta los efectos de la crisis del 2001. De igual manera, se focalizó en la relevancia de la producción colectiva, es decir, en un proceso desencadenado por los cruces y anudamientos deseantes entre miembros singulares. En tales coordenadas se encontró que el matiz lúdico habita los encuentros, ya que se trata de un espacio de sociabilidad en el que se improvisa con otros en las relaciones de intercambio que se establecen en un determinado grupo, cuya motivación pauta, incluso, los ritmos lógicos de su desarrollo.

\section{Palabras clave}

Micropolíticas, lazo social, invención, afecto, motivación.

* Doctora en Ciencias Sociales, Licenciada en Psicología y profesora de Enseñanza Media y Superior en Psicología en la Universidad de Buenos Aires. Es profesora del Postgrado Virtual sobre Construcción de Proyectos en Ciencias Sociales (CAICYT-CONICET) e investigadora en proyectos de SACyT, CEDES, Agencia-FONCYT y la Facultad de Ciencias Sociales del Instituto de Investigación Gino Germano de la Universidad de Buenos Aires. El presente artículo es producto de sus estudios en este instituto. Correo electrónico: karina.benito@speedy.com.ar 


\begin{abstract}
This paper presents the advances of a project of doctoral research in Social Sciences of the UBA. From a qualitative methodology spaces, clubs and cultural centers were explored in the city of Buenos Aires from the return of the democracy up to the effects of the crisis of 2001. The article focused in the relevancy of the collective production. It is a question of a process unleashed by the crossings of the desire between singular members. In such coordinates it was rerealed that the playful shade lives in the meetings, since it is a question of a space of sociableness where it is improvised with others in the relations hips of exchange between subjects of a certain group, whose motivation rules, even, the logical paces of its development.
\end{abstract}

\title{
Keywords
}

Micropolicies, social ties, invention, affection, motivation.

\section{Introducción}

La producción colectiva se caracteriza por un particular intercambio e interrelación con otros en dinámicas que, a su modo, son alegres, sin por ello perder la seriedad del asunto ni el motor implícito del entusiasmo colectivo. Resulta oportuno recordar que en el campo de las ciencias sociales algunos fenómenos sociales, tales como espacios, clubes o centros culturales autogestionados en momentos de crisis, si bien suelen ser explicados como provocados por causas vinculadas a los procesos de la globalización, las reformas estructurales, la terciarización, la precarización y la flexibilización laboral-experiencias analizadas en virtud de un trabajo empírico-, también son producto del amor fati. Es decir, a pesar de las vicisitudes que impone el destino, surgen también por el desafío de los sujetos al inventar colectivamente en determinadas circunstancias, incluso las adversas, y en eso radica el gusto compartido.

\section{De la diversión a la aparición de lo diverso: al acecho de una estructura armónica}

¿Cuál es el motivo por el que se fundan estos espacios para el encuentro con otros? ¿Por qué los colectivos gestan espacios, clubes o centros culturales, aun ante coyunturas críticas? ¿Qué posibilita que los sujetos se agrupen para contrarrestar los efectos de una crisis? ¿Cómo es posible que se elija un centro cultural como propuesta de amparo ante el quiebre de las protecciones clásicas? No resulta factible encontrar un correlato de sentido unívoco si se considera que en las dinámicas creativas emerge algo del orden de la diversión, y eso implica, tal como las raíces etimológicas de la palabra lo indican, la aparición de lo diverso.

Sin embargo, las diversiones cambian en las distintas épocas según el modo de convivencia de los hombres (Brecht, 1957), y quienes las emprenden le asignan su tiempo, dedicación y seriedad a tal asunto, además de un ingrediente de apasionamiento que los caracteriza (Deleuze, 1974). Según Spinoza 
(1980), las pasiones si bien son episódicas y precarias individuaciones de estados vividos, también son articuladoras de lo social, ya sea que se consoliden como una organización $u$ otra. Se trata de estados afectivos de fuerzas puestas en movimiento, es decir, existe una intensidad difícilmente mesurable, aunque condensa en sí la distinción entre quienes están “comprometidos" y quienes solo asisten como espectadores o consumidores. Por consiguiente, la participación no es subsumible a un producto que se compra en el mercado, porque el gusto se encuentra en determinados procesos de sociabilidad.

Dentro y fuera de esta constelación llamada "sociedad" se desarrolla una especial estructura sociológica correspondiente a las realidades del arte y del juego; estructura que toma su forma de estas, pero que sin embargo deja su realidad detrás de estas. Estimar en qué medida posee valor explicativo el concepto de "impulso lúdico" o "impulso artístico" es una cuestión abierta, pero, al menos, llama la atención el hecho de que todo juego o actividad artística incluye un elemento común no afectado por sus diferencias de contenido. Un cierto dejo de satisfacción se encuentra tanto en los juegos gimnásticos como en los de cartas, en la música o en el arte plástico; algo que no tiene nada que ver con que ambos sean artes y que tanto los juegos gimnásticos como los de cartas sean juegos. Un elemento común, una semejanza en la reacción y la necesidad psicológica se encuentra en todas esas cosas -algo fácilmente distinguible del propio interés especial que diferencia a cada uno-. En el mismo sentido, se puede hablar de un impulso del hombre hacia la sociabilidad (Simmel, 2002: 195-196).

El dejo de satisfacción, así como las tensiones características de los impulsos, domina los quehaceres, a modo de un ritmo interno que impone un dinamismo en los proyectos característicos del movimiento lúdico. El movimiento mantiene una relación especial con lo imperceptible - esa es su naturaleza-, pues la percepción solo puede captar el movimiento como la traslación de un móvil o el desarrollo de una forma. Los movimientos y los devenires, es decir, las puras relaciones de velocidad y lentitud, los puros afectos, están por debajo o por encima del umbral de percepción. Los umbrales de percepción son sin duda relativos; así, pues, siempre habrá uno capaz de escapar al otro: el ojo del águila... Pero, a su vez, el umbral adecuado solo podrá proceder en función de una forma perceptible y de un sujeto percibido, apercibido (Deleuze y Guattari, 2002: 282).

Se trata de movimientos con sus propias normas, ya sean prácticas con reglas explicitas u otras que el grupo descubre sobre la marcha como códigos implícitos que con el tiempo se enuncian; así, tales códigos atemperan lo que podría verse como un caos aparente. Aun cuando no existen reglas explícitas en los procesos -los amateurs construyen decálogos, manifiestos, proclamas e, incluso, formas regladas-, los participantes apuestan a determinadas construcciones, respetando códigos existentes. De este modo, los patrones de intercambio son avalados por los involucrados, ya que los respetan porque nacieron, generalmente, por dinámicas propias de trabajo, a partir de formas cooperativas y asociativas.

El anterior proceso primero se hace en entornos locales, donde hay individuos capaces de dividir y conectar actores y procesos cruciales, donde a esos grupos de individuos se les presta la posibilidad de separar y reunir algunas de sus experiencias, pero también de identificarse en tanto partes de una comunidad más vasta. Esta comunidad facilita la activación de una cierta interacción creativa y ofrece contextos en los cuales los participantes pueden establecer "acuerdos generales sobre procedimientos y resultados"; de igual 
manera, algunos pueden ponerse en posición de "árbitros que establecen límites a las actuaciones, la habilidad individual, el conocimiento"; también pueden proponerse formas de preparación disciplinada y acumularse las experiencias en historias (Ladagga, 2006).

En la gestación de estos espacios, clubes o centros culturales se buscó un formato, es decir, a través de pautas consensuadas y acuerdos se sostuvieron las tensiones que pautan los tiempos lógicos en los grupos. "En ese sentido, devenir todo el mundo es hacer del mundo un devenir, es crear una multitud, es crear un mundo, mundos, es decir, encontrar sus entornos y sus zonas de indiscernibilidad" (Deleuze y Guattari, 2002: 281).

Los improvisadores en diversas artes conocen la importancia de acuerdos consensuados en una atmósfera grupal, a modo de un orden que se suscita en el encuentro mismo. Así, por ejemplo, en el jazz conocen la importancia del clima alcanzado grupalmente y perciben los códigos implícitos básicamente porque saben que la ejecución de las interpretaciones no es a partir de la lectura de una fiel partitura, sino que la base está en la improvisación, en tanto que el intérprete recrea respetando una estructura armónica. La atmósfera grupal está en la interpretación más que en el compositor, diferenciándose así de la tradicional música clásica. Eso genera que el grupo se mantenga en atención ante la formación y los patrones que se dan rítmicamente en ese encuentro. Incluso en la improvisación en danza se crean partituras de movimiento que se gestan en la interacción con otros, en un determinado espacio y tiempo. Se trata de secuencias a través de un gesto lúdico en la interacción con otros, donde se compone en tiempo real. Es un gesto o una forma que adviene en torno a un gusto mutuo, sin seguir un procedimiento estrictamente reglado que paute las acciones, porque se conoce previamente el fin a alcanzar. En relación a tal dimensión,
Agamben (2001) enuncia, por ejemplo, que en la danza el gesto es, precisamente, porque no consiste en otra cosa que en soportar y exhibir los movimientos corporales. El gesto es la exhibición de una medialidad: el hacer visible el medio como tal.

En las experiencias analizadas, lo que queda en evidencia no es solo el marco en el que los amateurs sitúan sus propuestas, sino que también tornan legibles las circunstancias coyunturales, en tanto se exhibe la medialidad. De este modo, se enuncian los movimientos corporales, es decir, las intenciones de los sujetos en sus búsquedas quedan expuestas en sus gestos. Según Spinoza, el cuerpo humano dispone de una potencia de obrar dada, cuyo crecimiento se da por el modo en el que es afectado. Es decir, las afecciones humanas son impresiones de los cuerpos entre sí. Dicho de otro modo: el alma produce ideas y conoce, pero desde el soporte de un cuerpo, desde su medialidad, motivo por el cual la forma no es dada a priori sino a posteriori de una problemática común que interpela a la sensibilidad de un determinado colectivo. "Es decir, el gesto abre la esfera del ethos como esfera propia por excelencia de lo humano" (Agamben, 2001: 53).

En ese carácter ético, el gesto se entiende porque los valores se articulan no solo como un contenido relacionado con su forma, es decir, se estima el gesto como imbricado también en su proceso. En este sentido, se podría deducir, a partir del trabajo exploratorio realizado a través de las experiencias analizadas, que se exhiben los medios al igual que los instrumentos que utilizaba Galileo para medir los astros. ¿Por qué resulta relevante exhibir los medios? Tal vez porque se persiguen modos en los que cada logro alcanzado está articulado por el gusto mutuo ante la construcción de un esfuerzo colectivo. 


\section{La exhibición de una medialidad}

Las experiencias analizadas demuestran que se apela a la cultura para atemperar transformaciones sociales, con el fin de que algún aura se extienda a pesar de determinadas conmociones socio-históricas imbricadas. Lo relevante es que en una atmósfera grupal y en un estado de entusiasmo colectivo se la convoca para articular estructuras de relaciones entre sujetos en circunstancias disímiles. En las experiencias analizadas, las circunstancias se presentan como desfavorables, y se requiere del vínculo con otros como el revés de un trazo hilvanado delicadamente. En este sentido, la cultura aparece interviniendo en conflictividades sociales donde además causa su emergencia, porque ya se explicó que no está exenta de su entorno económico, histórico, político y social. "En el modo de producción capitalista, ni el arte ni la cultura en general pueden sobreponerse, superar las condiciones de producción a las que están sometidos. Lo que sí puede y debe hacer la cultura, en su constante autodestrucción, es mostrar esas condiciones de producción" (Arribas, 2007a: 1).

\section{Cultura, ¿proteger, cultivar, habitar y venerar?}

La raíz latina de la palabra cultura es colere, que puede significar desde cultivar y habitar, hasta veneración y protección; a través del latín cultus desemboca en culto. "Cultura" es un término que se encuentra en un umbral, porque encierra en sí mismo controversias que oscilan entre lo dado y lo creado, entre la tradición y la innovación. En el sentido de producción, la cultura evoca un control y, a la vez, un desarrollo espontáneo, de modo tal que es asequible porque lo cultural es lo que podemos transformar; sin embargo, el elemento que hay que alterar tiene su propia existencia autónoma. Así es que la cultura tiene reglas, se encuentra en ese umbral de interacción entre lo regulado y lo no regulado. La dimensión simbólica es intrínseca a la cultura, y a través de su función es posible articular una aplicación creativa de las mismas, mas no un determinismo. De esta manera, la propia categoría implica tensiones entre racionalidad y espontaneidad.

En la cultura siempre hay algo que la descentra, siempre hay una tensión o excentricidad como residuo de aquello inaprensible por el progreso o la racionalidad. Es simultánea a ella una conflictividad que la pone en su sitio y, a la vez, amenaza con desmantelarla. Por consiguiente, existen incongruencias que no remiten solo a una irregularidad de la planificación, un problema técnico, una grilla mal mesurada o el descuido de los expertos en la abstracción modélica de la burocracia y la gestión. No se trata tampoco de un exceso, desvío o accidente, sino que es el elemento constitutivo de la cultura y su apropiación por parte de la modernidad y su lógica racional. Las contradicciones del progreso se describieron en las experiencias, hasta el punto de explicar, incluso, las complejidades en las que el arte, en situaciones de fragilidad social, sobrelleva propuestas.

En este sentido, los colectivos atemperan las incoherencias de las conmociones sociales y de las ruinas que dejó la dictadura: el deterioro de lo público, la precariedad de la asociatividad y los dispositivos de desconfianza que socavaron las lógicas cooperativas. Buscan construir sentidos y también afectos, a fin de mitigar las incoherencias de la modernidad. Las experiencias culturales en coyunturas críticas demuestran la existencia de espacios en peligro de extinción mientras los grupos buscan nuevas ideas, nuevas formas posibles.

El buen método, decía Spinoza, no precede al conocimiento, es interior a él; la idea de la idea -la reflexión, el método- requiere partir de una idea verdadera, la cual es tanto 
signo de sí misma como de lo falso. Cuando se parte de ahí se trata de producir, de extraer consecuencias de ello y punto (indagaciones efectivas). Claro, esto no va de suyo: hay que trabajar sobre los conceptos bajo condiciones específicas para que algo material precipite, para que algo anude imprevistamente. Sin embargo, para que algo de ello acontezca es preciso interrumpir o suspender cualquier prescripción normativa, cualquier estándar fijo o protocolo de producción (Farrán, 2010: 92).

\section{Gestación de formatos creativos}

Las experiencias analizadas conforman espacios de sociabilidad, los cuales han sido delineados definiendo el valor del desarrollo de una confianza mutua. No solo se hace referencia a una delimitación geométrica, sino también a una localización simbólica para respirar "camaradería" incluso en tales espacios. Las experiencias reveladas resultan particulares porque se dan en una gestación colectiva en un formato creativo: una fábrica en quiebra que inaugura un centro cultural en su interior, un club que le da albergue a un emprendimiento asociativo entre varias asociaciones, como el Club Alemán, Asociación Belga en Buenos Aires, Club Francés, Asociación Holandesa, Club Danés, Club Sueco, Club Español, Asociación Austriaca, Círculo Italiano, los cuales, conmovidos por la crisis social, deciden aunar sus esfuerzos y conformar el Club Europeo o el Complejo Cultural Chacra de los Remedios. Este último es un espacio público abandonado que los vecinos reciclan pidiendo al Gobierno el reconocimiento de sus edificios como patrimonio histórico, luego del retorno de la democracia; un club que resurge a través de las asambleas de la terrible crisis del 2001 que acechó a la Argentina, además de las diversas troupes "independientes" que producen de un modo "autogestivo".
En las interacciones de tales propuestas hay territorio desde el momento en que hay expresividad del ritmo (Deleuze y Guattari, 2002), desde el momento en que hay relación entre el espacio existente y el posible, desde cuando los valores y las fuerzas morales no se conciben separados del transcurrir que gestan, sino que constituyen un vibrar al acecho de una estructura armónica. Esto, debido a que la micropolítica cuestiona lo que aparece aceptado como inmutable, es decir, aquello que se cree socialmente naturalizado, sin que se distinga que es producto de una construcción sociohistórica.

\section{Un efecto de distanciamiento, ¿podría ser de otro modo?}

Arribas (2007b) explica ideas centrales acuñadas por Brecht e identificables en Historias del Señor Keuner y Me-ti. Libro de los cambios, donde aparecen retratados dos tipos de conductores: uno aprendió perfectamente las reglas de conducción, las respeta y las aprovecha para abrirse camino entre el tráfico; otro maneja el coche colocándose mentalmente en la posición de los que marchan a su lado. A este segundo conductor le preocupa el tráfico en su conjunto: maniobra ante lo que se le cruza por el camino sin dejar de atender a lo que se le cruza al de adelante; logra su satisfacción cuando el tráfico fluye, sintiéndose una mínima parte integrante de él. ¿Una mínima parte? Brecht también la llama "unidad mínima", en tanto sus miembros no tienen por qué quererse entre sí, pues lo que quieren de verdad es el objetivo compartido. Por eso, no es cuestión de uno, ni de dos, ni de tres o cuatro, sino de eso tercero en movimiento que les une.

Ese movimiento es productivo-deseante, pues en un grupo se establece una relación productiva en torno a esa terceridad que lo vincula, de modo tal que se compromete entre sí, y sus integrantes son los responsables de 
ese quehacer gestado desde abajo. El grupo está advertido del contexto sociohistórico, en analogía a un tráfico que fluye y en el que se inscribe su ética, su estética y su unidad mínima. Los amateurs trabajan identificando la existencia de esa terceridad, en tanto persecución de un bien común que opera interviniendo en las experiencias analizadas respecto de lo naturalizado socialmente.

En este sentido, Arribas (2007b) enuncia, en relación a los conceptos de Brecht, que tampoco se trata de la desaparición de la división del trabajo. Dicho problema se ha encontrado como una tensión existente a cualquier forma burocrática y experta -característica del mundo desencantado-, motivo por el cual los amateurs cuestionan determinadas modalidades del saber técnico y del experto, porque en su especificidad profesional de gestión a veces olvidan la complejidad de ciertos asuntos, sin distinguir esa terceridad o complejidad del bien común. Entonces, la autora explica que todo depende de lo que se quiera decir con la división del trabajo y, en ese sentido, se podría pensar que un centro cultural, a veces, se centra en su programación porque esa es su tarea, aunque si hay un conflicto, se soluciona cuando el interrogante no se opaca respecto al bien común.

Lo anterior se debe al modo en el que se construyó, en sus inicios, cada espacio, ya que el conflicto era la existencia subyugada detrás de la construcción de sentido para el tratamiento específico, a través de la sociabilidad de una determinada propuesta cultural para atender a la problemática fundante. $L a$ tercera cosa de la cual habla Brecht resulta intimada por los amateurs, aunque a veces encarnen en sus experiencias el mismo conflicto que pretenden atender. Por ello, lo cotidiano se encuentra implícito en las condiciones de su existencia y, a veces, resulta tan obvio que demuestra el arbitrio y posibilidad de cambio. Se desprende de lo expuesto que los espacios, clubes y centros culturales obran como diagnóstico de situación que convoca a la reflexión, operando, a la vez, como terceridad del bien común e intervención colectiva.

\section{Referencias}

Agamben, G. (2001). Medios sin fin. Notas sobre la política. Valencia: Pretextos.

Arribas, S. (2007a, 28 y 29 de noviembre). Seminario Internacional: "Memoria e Industria Cultural”. Imagen-Aceleración-Digitalización. Madrid: IFS-CCHSCSIC. Disponible en http://www.proyectos.cchs.csic.es/fdh/sites/proyectos.cchs. csic.es.fdh/files/3SArribasImagenyautod estrucciondelacultura.pdf

Arribas, S. (2007b). Vivir en tercera persona. Revista Diagonal, 49. Disponible en http:/www.diagonalperiodico.net

Brecht, B. (1957). Brevario de estética teatral. Buenos Aires: La Rosada.

Deleuze, G. (1974). Spinoza, Kant, Nietzsche. Barcelona: Labor.

Deleuze, G. y Guattari, F. (2002). Mil mesetas. Valencia: Pretextos.

Farrán, R. (2010). La ley del deseo, esencia ética de lo político. Revista Isegoría, 42.

Ladagga, R. (2006). Estética de la emergencia. Buenos Aires: Adriana Hidalgo Editora.

Simmel, G. (2002). La sociabilidad. En Sobre la individualidad y las formas sociales. Buenos Aires: UNQUI.

Spinoza, B. (1980). Ética demostrada según el orden geométrico. Madrid: Ediciones Orbis. 